\title{
Switchable X-Ray Orbital Angular Momentum from an Artificial Spin Ice
}

\author{
Justin S. Woods $\odot,{ }^{1,2}$ Xiaoqian M. Chen, ${ }^{3,4}$ Rajesh V. Chopdekar $\odot,{ }^{3}$ Barry Farmer, ${ }^{1}$ Claudio Mazzoli $\odot,{ }^{5}$ \\ Roland Koch $\odot,{ }^{3}$ Anton S. Tremsin $\odot,{ }^{6}$ Wen Hu $\odot,{ }^{5}$ Andreas Scholl $\odot,{ }^{3}$ Steve Kevan, ${ }^{3}$ Stuart Wilkins, ${ }^{5}$ \\ Wai-Kwong Kwok®, ${ }^{2}$ Lance E. De Long $\odot,{ }^{1}$ Sujoy Roy $\odot,{ }^{3, *}$ and J. Todd Hastings $\oplus^{4, \dagger}$ \\ ${ }^{1}$ Department of Physics and Astronomy, University of Kentucky, Lexington, Kentucky 40506, USA \\ ${ }^{2}$ Materials Science Division, Argonne National Laboratory, Lemont, Illinois 60439, USA \\ ${ }^{3}$ Materials Sciences Division, Lawrence Berkeley National Laboratory, Berkeley, California 94720, USA \\ ${ }^{4}$ Department of Electrical and Computer Engineering, University of Kentucky, Lexington, Kentucky 40506, USA \\ ${ }^{5}$ National Synchrotron Light Source II, Brookhaven National Laboratory, Upton, New York 11973, USA \\ ${ }^{6}$ Space Sciences Laboratory, University of California, Berkeley, California 94720, USA
}

(Received 13 August 2020; accepted 23 December 2020; published 15 March 2021)

\begin{abstract}
Artificial spin ices (ASI) have been widely investigated as magnetic metamaterials with exotic properties governed by their geometries. In parallel, interest in X-ray photon orbital angular momentum (OAM) has been rapidly growing. Here we show that a square ASI with a patterned topological defect, a double edge dislocation, imparts OAM to scattered x rays. Unlike single dislocations, a double dislocation does not introduce magnetic frustration, and the ASI equilibrates to its antiferromagnetic (AFM) ground state. The topological charge of the defect differs with respect to the structural and magnetic order; thus, $\mathrm{x}$-ray diffraction from the ASI produces photons with even and odd OAM quantum numbers at the structural and AFM Bragg conditions, respectively. The magnetic transitions of the ASI allow the AFM OAM beams to be switched on and off by modest variations of temperature and applied magnetic field. These results demonstrate ASIs can serve as metasurfaces for reconfigurable x-ray optics that could enable selective probes of electronic and magnetic properties.
\end{abstract}

DOI: 10.1103/PhysRevLett.126.117201

Artificial spin ices (ASI) consist of patterned arrays of nanomagnets whose properties can be tuned based on geometry and competing interactions. As a result, ASIs are often designed to realize systems not readily accessible in nature $[1,2]$ such as geometrically frustrated magnetic lattices [3-5]. Frustration arising from topological defects, such as a single edge dislocation in a a square ASI [6], has also been studied. ASIs have the advantage that they can be reconfigured through a variety of field $[7,8]$, temperature $[9,10]$, and direct writing approaches [11,12]. Applications of ASIs have recently begun to emerge with a primary focus on computing [13-17]. Here we consider a square ASI with a previously unstudied topological defect, a double edge dislocation, shown in Fig. 1(a). Double edge dislocations are extremely rare defects in natural materials; however, they can be easily patterned into artificial systems. We demonstrate that such a modified ASI can impart OAM to $x$ rays in a controlled manner.

Published by the American Physical Society under the terms of the Creative Commons Attribution 4.0 International license. Further distribution of this work must maintain attribution to the author(s) and the published article's title, journal citation, and DOI.
OAM is a topological property of light for which the photon phase has a helical structure around its propagation axis [18-20]. Interest in optical OAM is increasing in the $\mathrm{x}$-ray regime where it could provide a selective probe of electronic and magnetic systems [21-29]. Recently, x-ray OAM was generated by gratings with structural topological defects [30]. However, the phase properties of coherently scattered photons from a 2D-magnetic lattice with a topological defect, as shown in Fig. 1(a), have not been studied in any detail. In fact, there are only very limited reports of magneto-optic effects associated with OAM beams at any wavelength [31,32]. Here we employ tunable, coherent, $\mathrm{x}$-ray sources to exploit the resonant enhancement of the spin-photon interaction cross section and observe soft x-ray OAM from ASIs.

To create an ASI with an edge dislocation one can displace the lattice points along one of the structural lattice vectors [33,34], taken as $\overrightarrow{\hat{x}}$ in Fig. 1(a), by a factor proportional to the azimuthal angle [35]. The Burgers vector that describes the double dislocation is $\vec{t}=2 a \overrightarrow{\hat{x}}$, where $a$ is the lattice constant. Thus, two additional structural periods are acquired over the course of a Burgers circuit; consequently, the defect has structural topological charge $\mathbb{Z}_{s}=2$, and we refer to this system as a "Z2-ASI." Equivalently, the structural lattice acquires a geometric, or Pancharatnam-Berry, phase of $4 \pi$ around a 
(a)

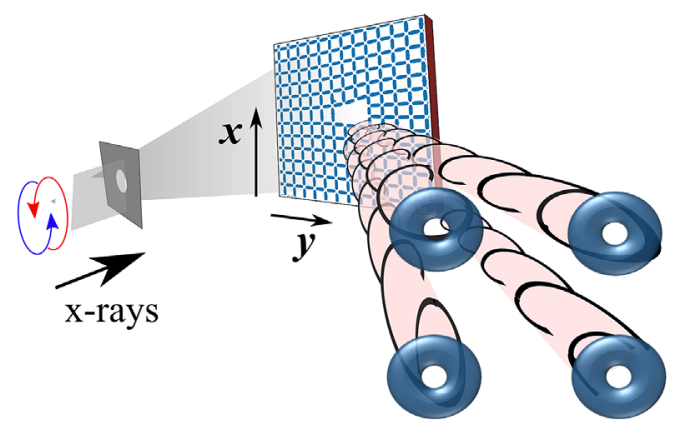

(b)

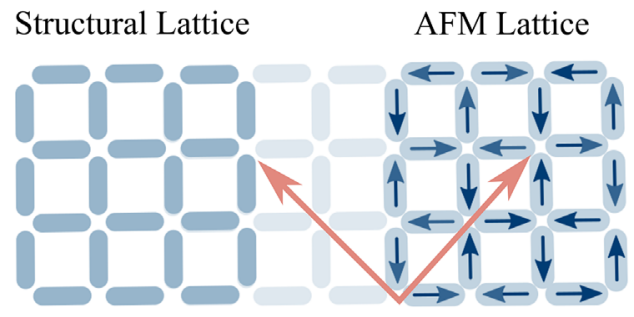

(c)

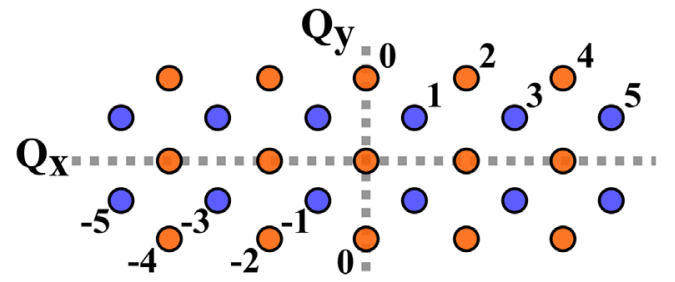

(d)

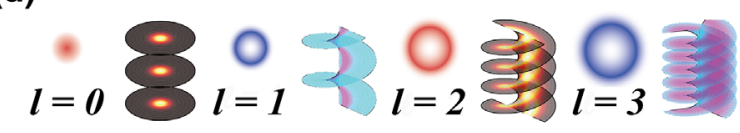

FIG. 1. Schematic of x-ray OAM from a topological defect in an artificial spin ice. (a) A square lattice of nanomagnets with a topological defect imparts orbital angular momentum to diffracted $x$ rays. The structural-lattice defect has topological charge 2 and generates even-order OAM in the charge-scattered $\mathrm{x}$-ray beams. The antiferromagnetic ground state defect has topological charge 1 and produces odd-order OAM in the magnetically scattered x-ray beams (four lowest-order beams are shown). The magnetically scattered beams can be manipulated by varying temperature or magnetic field. (b) Structural lattice and AFM lattice. Red arrows show that the magnetic lattice is rotated $45^{\circ}$ with respect to the structural lattice. (c) Positions of charge (red) and magnetic (blue) OAM peaks in reciprocal space labeled by OAM quantum number. (d) Schematic of the intensity and phase fronts of beams with OAM quantum number $\ell=0,1,2$, and 3 . Red and blue represent charge and magnetic scattered beams carrying even- and odd-order OAM, respectively.

Burgers circuit [36,37]. It is known that square ASIs without edge dislocations can equilibrate into the antiferromagnetic (AFM) ground state near room temperature if the nanomagnets have sufficiently small volume and appropriate spacing [7,10,38]. We adopted dimensions similar to those used in Ref. [10] under the assumption that the edge dislocation would not dramatically alter the equilibration process. The AFM lattice has twice the lattice constant of the structural lattice. Thus, the magnetic topological charge of the defect would be $\mathbb{Z}_{m}=1$, and the AFM lattice would acquire a geometric phase of $2 \pi$ around the Burgers circuit.

$\mathrm{X}$-ray diffraction from the Z2-ASI yields chargescattered beams at integer reciprocal lattice points from the structural lattice and at half-integer reciprocal lattice points from the AFM lattice. For a structural topological charge of 2, the charge- and magnetic-scattered beams are expected to carry even and odd OAM quantum numbers, respectively, as illustrated in Fig. 1(c). These beams can also be orthogonally polarized; thus, the Z2-ASI can create spatially separated beams of differing polarization and OAM quantum number as discussed in more detail below. Beams carrying OAM will form regardless of in-plane rotation of the ASI because the phase-singularity is fixed and there are always nanomagnets whose magnetization has a component along the beam direction. Moreover, square ASIs exhibit antiferromagnetic-to-paramagnetic (AFM-to-PM) transitions with temperature $[9,10,39]$ and antiferromagnetic-to-ferromagnetic (AFM-to-FM) transitions under applied fields [7]. The critical temperatures and reversal fields are governed by the size of and interactions among the nanomagnets along with the properties of the constituent material. As a result, we predicted that the magnetically scattered OAM beams should be sensitive to temperature and applied magnetic field.

To confirm these predictions, we fabricated the Z2-ASI from ferromagnetic permalloy $\left(\mathrm{Ni}_{0.80} \mathrm{Fe}_{0.20}\right)$ using electronbeam lithography and lift off [35]. The resulting structure is shown in Fig. 2(a). The permalloy nanomagnets mimic Ising spins due to their large shape anisotropy. We chose the thickness of the permalloy to be between 2 and $3 \mathrm{~nm}$ so that the structure is thermally active and undergoes an AFM-to-PM transition near room temperature $\left(T_{N} \approx 300 \mathrm{~K}\right)$.

Similar structures without topological defects have been studied, and are known to order into an AFM ground state $[7,38,40,41]$. The addition of a single dislocation introduces topological frustration and prevents the lattice from reaching a single-domain AFM state [6]. However, for the double dislocation studied here, the magnetic lattice is not frustrated at the nearest-neighbor level. Thus, thermal fluctuations near the AFM-to-PM phase transition should enable the sample to attain a single-domain AFM ground state when slowly cooled below $T_{N}$ [10]. However, it was not known a priori whether the lattice distortion around the double edge dislocation would nucleate and/or pin superdomain walls or introduce longer nanomagnets that may not be thermally active [35]. These effects could prevent long-range ground state ordering despite the absence of direct magnetic frustration.

To resolve these questions, we used x-ray magnetic circular dichroism photoemission electron microscopy (XMCD-PEEM) to experimentally image the ground state 


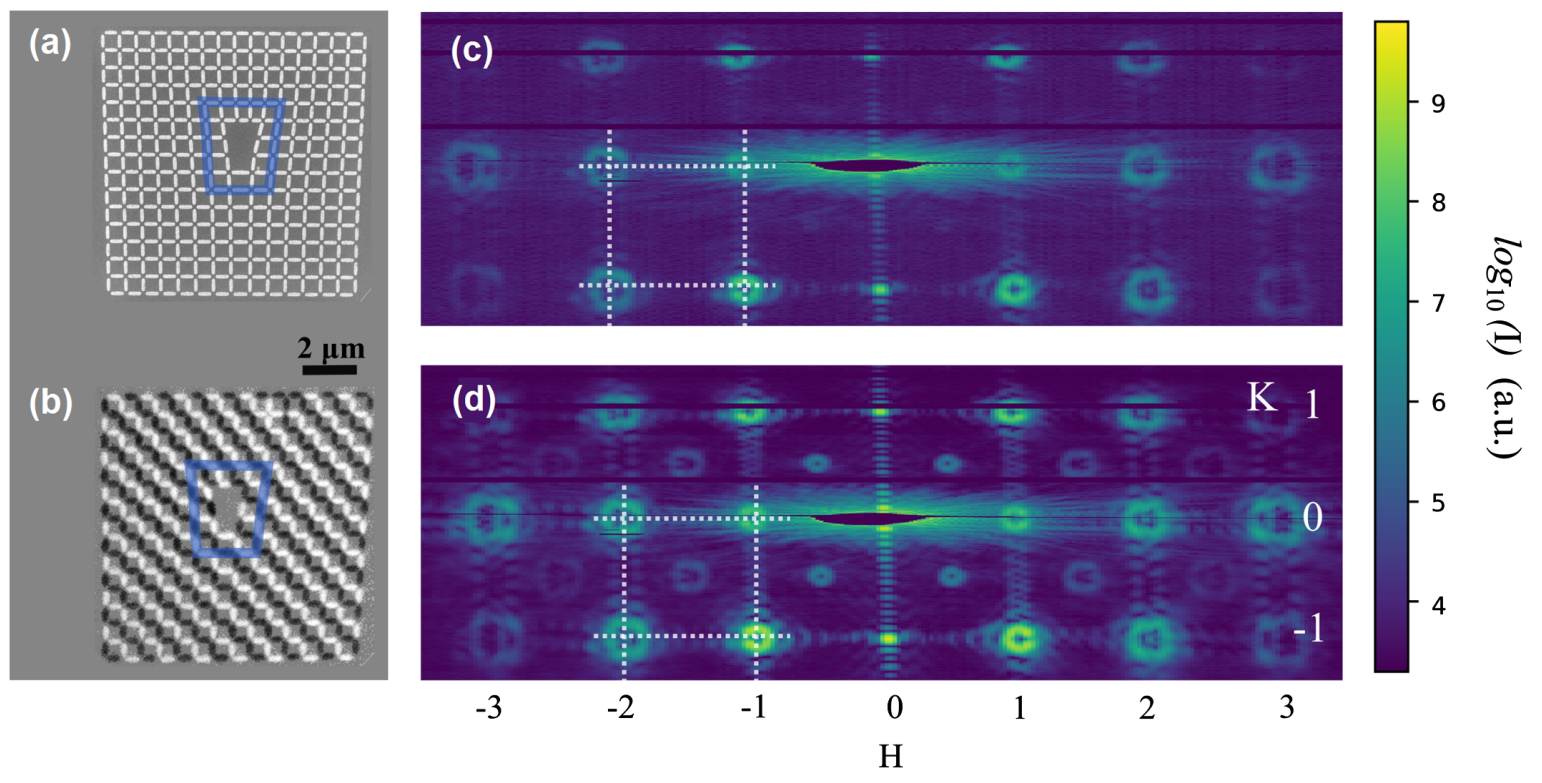

FIG. 2. Experimental realization of a single-domain AFM ground state in the Z2-ASI and resulting X-ray diffraction. (a) Scanning electron micrograph of a permalloy lattice of nanomagnets with a topological defect consisting of a double edge dislocation. (b) X-ray magnetic circular dichroism PEEM micrograph revealing the AFM ground state order. The blue boxes trace out a Burgers circuit. (c) Off-resonance diffraction produces x-ray vortex beams carrying even order orbital angular momentum at the structural Bragg conditions. (d) Diffraction at the resonance condition dramatically enhances vortex beams at the AFM Bragg conditions (half-integer values of $H$ and $K$ ) carrying odd-order OAM.

order as shown in Fig. 2(b). XMCD-PEEM is a magnetic imaging technique that probes magnetization parallel or antiparallel to the incident $\mathrm{x}$-ray beam. Magnetic images were recorded using the PEEM-3 microscope at beam line 11.0.1.1 of the Advanced Light Source. The incident x rays were tuned to the $\mathrm{Fe} L_{3}$ absorption edge $(\sim 708 \mathrm{eV})$, and the difference between the right- and left-circularly polarized $\mathrm{x}$-ray images was taken to emphasize magnetic contrast over chemical or topographic contrast.

The image in Fig. 2(b) was taken at $T=110 \mathrm{~K}$, well below the AFM-to-PM transition temperature. The $\mathrm{x}$-ray beam was oriented along the $[-11]$ direction of the structural lattice at $30^{\circ}$ grazing incidence. This sample orientation gives sensitivity to in-plane magnetic moments for both [10] and [01] oriented islands in the lattice. The white and black regions signify opposite magnetization directions of the nanomagnets, and confirm that the sample orders into an AFM ground state. A Burgers circuit drawn around the defect, indicated in blue in Figs. 2(a) and 2(b), shows that the structural lattice has two extra periods $\left(\mathbb{Z}_{s}=2\right)$ while the magnetic lattice has one extra period $\left(\mathbb{Z}_{m}=1\right)$ as discussed above. Thus, the engineered defect provides the desired topological charge and does not prevent single-domain ground state ordering.

With the antiferromagnetic ground state established, we conducted coherent $\mathrm{x}$-ray scattering experiments to study the OAM of the diffracted beams. X-ray scattering was conducted at the COSMIC-Scattering beam line at the Advanced Light Source, Lawrence Berkeley National
Laboratory, and at the Coherent Soft X-ray (CSX) beam line at the National Sychrotron Light Source II, Brookhaven National Laboratory. The diffracted photons were detected using a Timepix [42-44] based soft x-ray detector at ALS and fast CCD detector at CSX. The diffraction experiment was performed in a reflection geometry as shown in Fig. 1(a). A coherent beam of $\mathrm{x}$ rays was incident on the sample at $\theta=9^{\circ}$.

To obtain magnetic sensitivity, we employed resonant magnetic scattering by tuning the energy of the incident $x$ ray to $\mathrm{Fe} L_{3}$ edge $(708 \mathrm{eV})$. Figures 2(c) and 2(d) show the diffraction patterns from the Z2-ASI with $\mathrm{x}$ rays tuned away from $(690 \mathrm{eV})$ and on $(708 \mathrm{eV})$ the $\mathrm{Fe} L_{3}$ absorption edge, respectively. Vortex beams, bright rings with zero intensity at the center, appear at the structural Bragg condition, integral values of $(H, K)=\left[Q_{x} /(2 \pi / a), Q_{y} /(2 \pi / a)\right]$, for both off- and on-resonant x-ray illumination. On resonance, additional vortex beams become visible due to magnetic scattering at the charge-forbidden (half-integer) AFM Bragg condition [Fig. 2(d)]. Magnetic peaks have been previously observed from the AFM ground state of square lattices without topological defects [10,45], but here the topological defect yields vortex beams at the AFM Bragg condition.

The detailed characteristics, and especially the intensities, of these diffracted $\mathrm{x}$-ray beams are determined by the structure and form factors of the system, which are determined by the size, shape, and composition of the nanomagnets. However, the photon OAM quantum 
numbers and basic vortex nature of the beams can be found simply from the lattice sum, which, for a 2D square lattice with an edge dislocation, is given by

$$
L=\sum_{m} \exp \left(i \vec{Q} \cdot \overrightarrow{R_{m}}+i \vec{Q} \cdot \vec{t} \frac{\psi_{m}}{2 \pi}\right)
$$

where $\overrightarrow{R_{m}}$ are the lattice vectors of the undistorted lattice, $\vec{t}$ is the Burgers vector for the structural lattice, and $\psi_{m}$ is the azimuthal angle for the $m^{\text {th }}$ lattice vector [33]. Near a reciprocal lattice point $\vec{Q}_{0}$, this sum can be approximated as [35]

$$
L^{\prime}\left(\rho^{\prime}, \phi^{\prime}\right)=i^{\ell} \exp \left(i \ell \phi^{\prime}\right) U\left(\ell, \rho^{\prime}\right) .
$$

The diffracted beam resulting from the lattice sum in Eq. (2) is described by a phase factor $\exp \left(i \ell \phi^{\prime}\right)$ and a purely real amplitude $U\left(\ell, \rho^{\prime}\right)$. The phase factor reveals that any beam with $\ell \neq 0$ carries an orbital angular momentum of $\hbar \ell$ [18], where the integer $\ell$ is given by

$$
\ell=\frac{\overrightarrow{Q_{0}} \cdot \vec{t}}{2 \pi}
$$

As noted above, charge-scattered beams occur at integral valued reciprocal lattice vectors. For the case of $\vec{t}=2 a \overrightarrow{\hat{x}}$ considered here, Eq. (3) yields $\ell=2 H$, where $H=Q_{x} / 2 \pi=1,2,3, \ldots$ and $\ell$ is always even. Antiferromagnetically scattered beams occur at halfinteger reciprocal lattice vectors where $H=Q_{x} / 2 \pi=$ $1 / 2,3 / 2,5 / 2, \ldots$, and $\ell$ is odd. Thus, the diffracted beams from the structural lattice carry even-order angular momentum (i.e., $0 \hbar, 2 \hbar, 4 \hbar, \ldots$ ) consistent with $\mathbb{Z}_{s}=\mathbb{N}=2$. The magnetically diffracted beams carry odd-order angular momentum (i.e., $1 \hbar, 3 \hbar, 5 \hbar, \ldots$ ). This is consistent with $\mathbb{Z}_{m}=1$ because scattering with even-order angular momentum is forbidden by the symmetry of the AFM structure factor. The amplitude in Eq. (2), $U\left(\ell, \rho^{\prime}\right)$, reveals that the beams that carry OAM $(\ell \neq 0)$ will exhibit a vortex structure. The amplitude also indicates that the vortex radius will increase with $\ell$ [35]. All of these results are consistent with the diffraction patterns shown in Figs. 2(c) and 2(d).

The polarization sensitivity of x-ray scattering [46] also enables direct determination of the phase progression of the OAM beams and thus the OAM quantum numbers. Specifically, $\sigma$-polarized incident $\mathrm{x}$ rays yield charge scattered beams carrying even OAM with $\sigma$ polarization and magnetically scattered beams carrying odd OAM with $\pi$ polarization [35]. For circularly polarized incident light, there is interference between the charge and magnetically scattered components. These interference effects are most easily observed in the difference in scattered intensity between left- and right- $\left(I_{c+}\right.$ and $\left.I_{c-}\right)$ circularly polarized illumination, as shown in Fig. 3. The circular-polarization
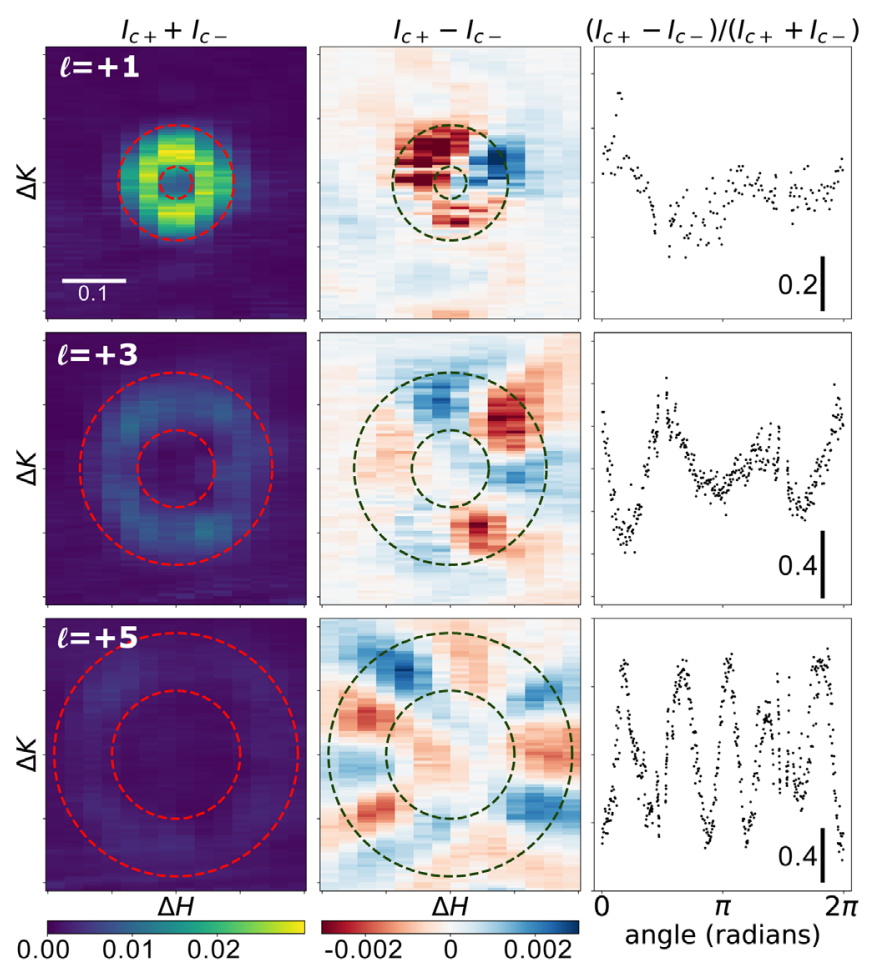

FIG. 3. Determination of the AFM OAM quantum number from interference between charge and magnetically scattered $\mathrm{x}$ rays. The sum and difference of diffracted intensities (arb. units) from left- $\left(I_{c+}\right)$ and right- $\left(I_{c-}\right)$ circularly polarized illumination are plotted for magnetic beams with $\ell=+1, \ell=+3$, and $\ell=+5$ OAM quantum numbers. The first column, $I_{c+}+I_{c-}$, shows the vortex structure of the beams. The second column, $I_{c+}-I_{c-}$, reveals the interference pattern between charge and magnetically scattered x rays. Dashed lines serve as a guide and the scale bar applies to all panels. Within the vortex ring, the number of interference fringes corresponds to the OAM quantum number as it would for interference with a plane wave. The third column plots $I_{c+}-I_{c-} / I_{c+}+I_{c-}$ versus azimuthal angle which reveals an approximately sinusoidal oscillation consistent with the OAM phase progression.

sensitivity arises from the phase difference in the $\pi$ component of left- and right-circularly polarized incident light. The interference patterns in Fig. 3 exhibit modulation of the intensity in the azimuthal direction about the axis of propagation. This is a result of the azimuthal phase progression of the primary beam with respect to the slow (or zero) phase progression in the tails of nearby chargescattered beams. The increasing number of fringes with diffracted order is consistent with increasing OAM and thus an increasing number of phase windings [47,48]. Plotting $I_{c+}-I_{c-}$ vs azimuthal angle for the first three AFM beams gives the number of fringes as $\ell=1,3$, and 5 which is the same as the predicted OAM quantum number from Eq. (3).

Finally, the temperature and magnetic field dependence of the AFM order provides control of the AFM-scattered OAM beams. Similar permalloy square lattices without topological defects undergo AFM-to-PM phase transitions 


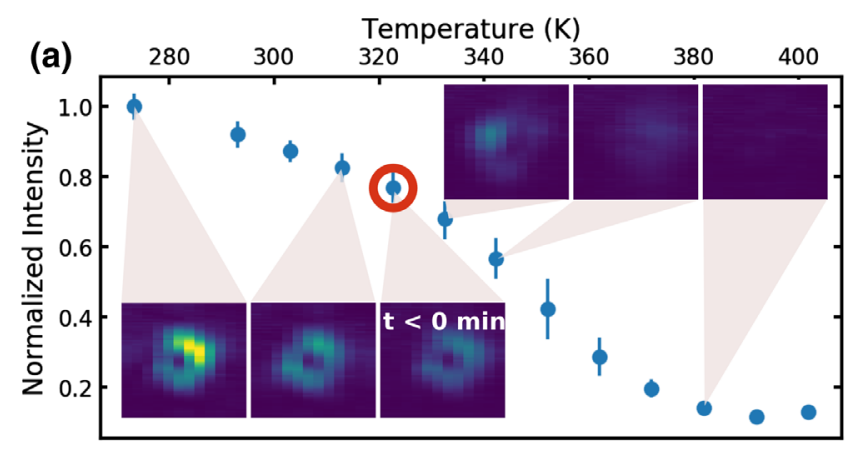

(b) Recovery of OAM

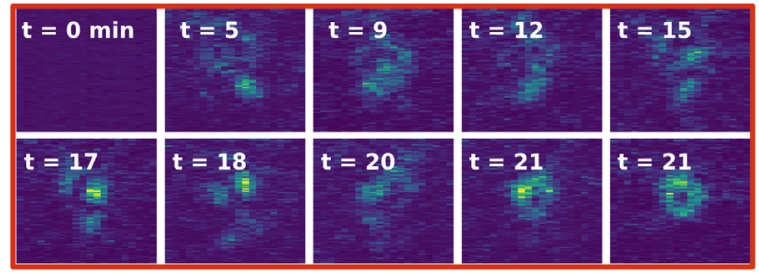

FIG. 4. Response of the Z2-ASI to temperature and applied magnetic field. (a) Temperature dependent x-ray intensity of the magnetically scattered OAM beam with $\ell=1$. As the sample approaches the antiferromagnetic-to-paramagnetic transition temperature $\left(T_{N} \approx 380 \mathrm{~K}\right)$ of the artificial lattice, the integrated intensity near the AFM Bragg condition decreases. (b) Time dependence of intensity after the beams are switched off using a magnetic field. The field is removed at $t=0$ and the temperature is held at $320 \mathrm{~K}$. The intensity for $t<0$ is labeled in (a). The speckle pattern at early time points indicates that disordered AFM domains form first. Afterwards, the sample further relaxes to its AFM ground state and the OAM vortex beam is restored. Indicated times are in minutes.

near room temperature [10] and AFM-to-FM transitions under applied magnetic field [7]. As temperature increases toward the AFM-to-PM transition of our Z2-ASI, the intensities of the OAM beams at the AFM Bragg condition weaken until they are extinguished above $T_{N} \approx 380 \mathrm{~K}$ as shown in Fig. 4(a). This result was replicated using a sample with lower $T_{N}$ [35].

If we apply an in-plane magnetic field to the sample, instituting an AFM-to-FM transition, the AFM OAM beams are also extinguished, as shown in the first frame of Fig. 4(b). In this case, we applied the field along the [1 0$]$ direction which was orthogonal to the beam axis. If we maintain the sample temperature close to $T_{N}$ and remove the magnetic field, the sample relaxes into disordered AFM superdomains within seconds as evidenced by the speckle patterns at short timescales of Fig. 4(b). In the future, these speckle patterns could be employed to characterize the outof-equilibrium dynamics of ASIs with topological defects. The sample relaxes to the AFM ground state over several minutes, as shown in the last frame of Fig. 4(b). A video of the fluctuation and relaxation processes is available in the Supplemental Material [35]. At the timescales measured here, relaxation is dominated by domain wall nucleation, annihilation, and transport which could be jammed or pinned. However, the relative contributions of these mechanisms is yet to be established. Regardless, the AFM OAM beams can clearly be switched on and off with small changes in temperature and applied field. ASIs with ground states that naturally contain phase progressions, such as finite-sized pinwheel tilings, will also be responsive to temperature and field and could be considered for controllable OAM generation.

We have shown that introducing a double edge dislocation in a square artificial spin ice does not prevent equilibration to a single-domain antiferromagnetic ground state. Thus, the patterned topological defect exhibits a structural charge of two and a magnetic charge of one. As a result, the ASI imparts even- and odd-order orbital angular momentum to charge- and magnetically scattered $\mathrm{x}$-ray photons, respectively. We determined the OAM quantum numbers of the magnetically scattered beams using a novel self-interference technique which exploits the polarization dependence of the resonant $\mathrm{x}$-ray scattering. Finally, the ASI's AFM-to-FM and AFM-to-PM transitions allowed the AFM OAM beams to be switched on- and off- with modest changes in field and temperature, respectively. These findings represent a first step toward realizing reconfigurable optics for the generation and analysis of soft x-ray orbital angular momentum. More broadly, these studies show that engineering defects in nanoscale magnetic lattices offers a powerful tool for designing $\mathrm{x}$-ray metasurfaces.

The work is supported by the U.S. Department of Energy, Office of Science, Office of Basic Energy Sciences under Award No. DE-SC0016519. The work used Timepix based soft x-ray detector, development of which is supported by DOE through award RoyTimepixDetector. X. M. C. and S. D. K. acknowledge partial support through DOE BES DE-AC02-05-CH11231 within the Nonequilibrium Magnetic Materials Program (MSMAG). This research used resources of the Advanced Light Source, which is a DOE Office of Science User Facility under Contract No. DE-AC02-05CH11231. This research used the 23-ID-1 (CSX) beamline of the National Synchrotron Light Source II, a U.S. Department of Energy (DOE) Office of Science User Facility operated for the DOE Office of Science by Brookhaven National Laboratory under Contract No. DE-SC0012704. Use of the Center for Nanoscale Materials, an Office of Science user facility, was supported by the U.S. Department of Energy, Office of Science, Office of Basic Energy Sciences, under Contract No. DE-AC02-06CH11357. Sample characterization was performed in the Material Science Division at Argonne National laboratory and supported by the U.S. Department of Energy, Office of Science, Basic Energy Sciences, Materials Sciences and Engineering Division. This work was performed in part at the University of Kentucky Center for Nanoscale Science and Engineering and Center for Advanced Materials, 
members of the National Nanotechnology Coordinated Infrastructure (NNCI), which is supported by the National Science Foundation (NNCI-2025075).

J. W. and X. C contributed equally to the work.

*sroy@lbl.gov

†todd.hastings@uky.edu

[1] S. H. Skjærvø, C. H. Marrows, R. L. Stamps, and L. J. Heyderman, Advances in artificial spin ice, Nat. Rev. Phys. 2, 13 (2020).

[2] C. Nisoli, R. Moessner, and P. Schiffer, Colloquium: Artificial spin ice: Designing and imaging magnetic frustration, Rev. Mod. Phys. 85, 1473 (2013).

[3] Y. Perrin, B. Canals, and N. Rougemaille, Extensive degeneracy, Coulomb phase and magnetic monopoles in artificial square ice, Nature (London) 540, 410 (2016).

[4] A. Farhan, M. Saccone, C. F. Petersen, S. Dhuey, R. V. Chopdekar, Y.-L. Huang, N. Kent, Z. Chen, M. J. Alava, T. Lippert, A. Scholl, and S. v. Dijken, Emergent magnetic monopole dynamics in macroscopically degenerate artificial spin ice, Sci. Adv. 5, eaav6380 (2019).

[5] I. Gilbert, Y. Lao, I. Carrasquillo, L. OBrien, J. D. Watts, M. Manno, C. Leighton, A. Scholl, C. Nisoli, and P. Schiffer, Emergent reduced dimensionality by vertex frustration in artificial spin ice, Nat. Phys. 12, 162 (2016).

[6] J. Drisko, T. Marsh, and J. Cumings, Topological frustration of artificial spin ice, Nat. Commun. 8, 14009 (2017).

[7] A. Farhan, P. M. Derlet, A. Kleibert, A. Balan, R. V. Chopdekar, M. Wyss, J. Perron, A. Scholl, F. Nolting, and L. J. Heyderman, Direct Observation of Thermal Relaxation in Artificial Spin Ice, Phys. Rev. Lett. 111, 057204 (2013).

[8] J. Sklenar, Y. Lao, A. Albrecht, J. D. Watts, C. Nisoli, G.-W. Chern, and P. Schiffer, Field-induced phase coexistence in an artificial spin ice, Nat. Phys. 15, 191 (2019).

[9] O. Sendetskyi, V. Scagnoli, N. Leo, L. Anghinolfi, A. Alberca, J. Luning, U. Staub, P. M. Derlet, and L. J. Heyderman, Continuous magnetic phase transition in artificial square ice, Phys. Rev. B 99, 214430 (2019).

[10] X. M. Chen, B. Farmer, J. S. Woods, S. Dhuey, W. Hu, C. Mazzoli, S. B. Wilkins, R. V. Chopdekar, A. Scholl, I. K. Robinson, L. E. De Long, S. Roy, and J. T. Hastings, Spontaneous Magnetic Superdomain Wall Fluctuations in an Artificial Antiferromagnet, Phys. Rev. Lett. 123, 197202 (2019).

[11] Y.-L. Wang, Z.-L. Xiao, A. Snezhko, J. Xu, L. E. Ocola, R. Divan, J. E. Pearson, G. W. Crabtree, and W.-K. Kwok, Rewritable artificial magnetic charge ice, Science 352, 962 (2016).

[12] J. C. Gartside, D. M. Arroo, D. M. Burn, V. L. Bemmer, A. Moskalenko, L. F. Cohen, and W. R. Branford, Realization of ground state in artificial kagome spin ice via topological defect-driven magnetic writing, Nat. Nanotechnol. 13, 53 (2018).

[13] H. Arava, P. M. Derlet, J. Vijayakumar, J. Cui, N. S. Bingham, A. Kleibert, and L. J. Heyderman, Computational logic with square rings of nanomagnets, Nanotechnology 29, 265205 (2018).
[14] H. Arava, N. Leo, D. Schildknecht, J. Cui, J. Vijayakumar, P. M. Derlet, A. Kleibert, and L. J. Heyderman, Engineering Relaxation Pathways in Building Blocks of Artificial Spin Ice for Computation, Phys. Rev. Applied 11, 054086 (2019).

[15] E. Iacocca, S. Gliga, and O. G. Heinonen, Tailoring SpinWave Channels in a Reconfigurable Artificial Spin Ice, Phys. Rev. Applied 13, 044047 (2020).

[16] S. Gliga, E. Iacocca, and O. G. Heinonen, Dynamics of reconfigurable artificial spin ice: Toward magnonic functional materials, APL Mater. 8, 040911 (2020).

[17] J. H. Jensen and G. Tufte, Reservoir computing in artificial spin ice, Artificial Life Conf. Proc. 32, 376 (2020).

[18] L. Allen, M. W. Beijersbergen, R. J. C. Spreeuw, and J. P. Woerdman, Orbital angular momentum of light and the transformation of Laguerre-Gaussian laser modes, Phys. Rev. A 45, 8185 (1992).

[19] G. Molina-Terriza, J. P. Torres, and L. Torner, Twisted photons, Nat. Phys. 3, 305 (2007).

[20] Y. Shen, X. Wang, Z. Xie, C. Min, X. Fu, Q. Liu, M. Gong, and $\mathrm{X}$. Yuan, Optical vortices 30 years on: OAM manipulation from topological charge to multiple singularities, Light Sci. Appl. 8, 90 (2019).

[21] L. Loetgering, M. Baluktsian, K. Keskinbora, R. Horstmeyer, T. Wilhein, G. Schütz, K. S. E. Eikema, and S. Witte, Generation and characterization of focused helical x-ray beams, Sci. Adv. 6, eaax8836 (2020).

[22] L. Rego, K. M. Dorney, N. J. Brooks, Q. L. Nguyen, C.-T. Liao, J. S. Román, D. E. Couch, A. Liu, E. Pisanty, M. Lewenstein, L. Plaja, H. C. Kapteyn, M. M. Murnane, and C. Hernández-García, Generation of extreme-ultraviolet beams with time-varying orbital angular momentum, Science 364, eaaw9486 (2019).

[23] M. van Veenendaal and I. McNulty, Prediction of Strong Dichroism Induced by X Rays Carrying Orbital Momentum, Phys. Rev. Lett. 98, 157401 (2007).

[24] M. van Veenendaal, Interaction between x-ray and magnetic vortices, Phys. Rev. B 92, 245116 (2015).

[25] J. Vila-Comamala, A. Sakdinawat, and M. Guizar-Sicairos, Characterization of $\mathrm{x}$-ray phase vortices by ptychographic coherent diffractive imaging, Opt. Lett. 39, 5281 (2014).

[26] E. Hemsing, A. Knyazik, M. Dunning, D. Xiang, A. Marinelli, C. Hast, and J. B. Rosenzweig, Coherent optical vortices from relativistic electron beams, Nat. Phys. 9, 549 (2013).

[27] A. Sakdinawat and Y. Liu, Soft-x-ray microscopy using spiral zone plates, Opt. Lett. 32, 2635 (2007).

[28] A. G. Peele, P. J. McMahon, D. Paterson, C. Q. Tran, A. P. Mancuso, K. A. Nugent, J. P. Hayes, E. Harvey, B. Lai, and I. McNulty, Observation of an x-ray vortex, Opt. Lett. 27, 1752 (2002).

[29] D. Cojoc, B. Kaulich, A. Carpentiero, S. Cabrini, L. Businaro, and E. Di Fabrizio, X-ray vortices with high topological charge, Microelectronic Eng. Micro- Nano-Eng. MNE 2005, 83, 1360 (2006).

[30] J. C. T. Lee, S. J. Alexander, S. D. Kevan, S. Roy, and B. J. McMorran, LaguerreGauss and HermiteGauss soft X-ray states generated using diffractive optics, Nat. Photonics 13, 205 (2019).

[31] J. A. Davis, E. Carcole, and D. M. Cottrell, Intensity and phase measurements of nondiffracting beams generated 
with a magneto-optic spatial light modulator, Appl. Opt. 35, 593 (1996).

[32] N. A. Groshenko, O. S. Makalish, and A. V. Volyar, Optical vortices in the scattering field of magnetic domain holograms, Tech. Phys. 43, 1450 (1998).

[33] A.J.C. Wilson, A note on diffraction by a dislocation, Research 3, 387 (1950).

[34] B. T. M. Willis, An optical method of studying the diffraction from imperfect crystals. II. Crystals with dislocations, Proc. R. Soc. Ser. A 239, 192 (1957).

[35] See Supplemental Material at http://link.aps.org/ supplemental/10.1103/PhysRevLett.126.117201 for S1 for more detail concerning the square lattice with an edge dislocation and its fabrication, S2 for more detail concerning the vortex beams generated by the Z2-ASI, S3 for more detail concerning the polarization of the vortex beams, S4 for temperature dependent x-ray diffraction results from a sample with a lower transition temperature, and S5 the online video for more detail about relaxation to the AFM ground state after application of an in-plane magnetic field.

[36] J. F. Nye, M. V. Berry, and F. C. Frank, Dislocations in wave trains, Proc. R. Soc. A 336, 165 (1974).

[37] E. Cohen, H. Larocque, F. Bouchard, F. Nejadsattari, Y. Gefen, and E. Karimi, Geometric phase from AharonovBohm to PancharatnamBerry and beyond, Nat. Rev. Phys. 1, 437 (2019).

[38] J. P. Morgan, A. Stein, S. Langridge, and C. H. Marrows, Thermal ground-state ordering and elementary excitations in artificial magnetic square ice, Nat. Phys. 7, 75 (2011).

[39] V. Kapaklis, U. B. Arnalds, A. Harman-Clarke, E. T. Papaioannou, M. Karimipour, P. Korelis, A. Taroni, P. C. W. Holdsworth, S. T. Bramwell, and B. Hjrvarsson, Melting artificial spin ice, New J. Phys. 14, 035009 (2012).

[40] G. Moller and R. Moessner, Artificial Square Ice and Related Dipolar Nanoarrays, Phys. Rev. Lett. 96, 237202 (2006).
[41] S. Zhang, I. Gilbert, C. Nisoli, G.-W. Chern, M. J. Erickson, L. OBrien, C. Leighton, P. E. Lammert, V. H. Crespi, and P. Schiffer, Crystallites of magnetic charges in artificial spin ice, Nature (London) 500, 553 (2013).

[42] A. S. Tremsin, J. V. Vallerga, and O. H. W. Siegmund, Overview of spatial and timing resolution of event counting detectors with microchannel plates, Nucl. Instrum. Methods Phys. Res., Sect. A 949, 162768 (2020).

[43] A. S. Tremsin, J. V. Vallerga, and R. R. Raffanti, Optimization of spatial resolution and detection efficiency for photon/electron/neutron/ion counting detectors with microchannel plates and quad timepix readout, J. Instrum. 13, C11005 (2018).

[44] M. Fiorini, J. Alozy, M. Bolognesi, M. Campbell, A. C. Ramusino, X. LLopart, T. Michel, S. F. Schifano, A. Tremsin, and J. Vallerga, Single-photon imaging detector with (10) ps timing and sub- $10 \mu \mathrm{m}$ position resolutions, J. Instrum. 13, C12005 (2018).

[45] J. Perron, L. Anghinolfi, B. Tudu, N. Jaouen, J.-M. Tonnerre, M. Sacchi, F. Nolting, J. Lüning, and L. J. Heyderman, Extended reciprocal space observation of artificial spin ice with $\mathrm{x}$-ray resonant magnetic scattering, Phys. Rev. B 88, 214424 (2013).

[46] J. P. Hill and D. F. McMorrow, X-ray resonant exchange scattering: Polarization dependence and correlation function, Acta Crystallogr. Sect. A 52, 236 (1996).

[47] A. White, C. Smith, N. Heckenberg, H. RubinszteinDunlop, R. McDuff, C. Weiss, and C. Tamm, Interferometric measurements of phase singularities in the output of a visible laser, J. Mod. Opt. 38, 2531 (1991).

[48] S. Pan, C. Pei, S. Liu, J. Wei, D. Wu, Z. Liu, Y. Yin, Y. Xia, and J. Yin, Measuring orbital angular momentums of light based on petal interference patterns, OSA Continuum 1, 451 (2018). 